\title{
A Pipeline Embolization Device for Original the Treatment of Large and Giant Intracranial Aneurysms: Initial Experience at a Single Center
}

Ryuta Nakae, Kensuke Suzuki, Koji Hirata, Yosuke Kawamura, Ryotaro Suzuki, Nobuyuki Shimizu, Masaya Nagaishi, Yoshihiro Tanaka, and Akio Hyodo

Objective: The Pipeline Embolization Device (PED) (Covidien, Irvine, CA, USA) is an endovascular microcatheter delivery system for the curative reconstruction of parent arteries harboring large/giant, wide-necked, and fusiform intracranial aneurysms. Here, we describe our initial experience with the PED to evaluate its safety, complications, and effectiveness. Methods: We retrospectively reviewed 23 consecutive patients who had received PED endovascular treatment of 23 intracranial aneurysms of the internal carotid artery between the petrous and superior hypophyseal segments between November 2015 and August 2016.

Results: A total of 11 complications were experienced by nine patients with a mean follow-up period of 6.5 months. Five major complications defined as ipsilateral stroke in four patients were encountered: two procedure-related ischemic strokes, one delayed ischemic stroke, and two intracerebral hemorrhages. In one patient, the symptom was persistent 3 months post-procedure. Of the other five patients with complications, four went on to recover completely 3 months post-procedure. Overall, the total complication rate for treatment with PED was 39.1\% (9/23). Morbidity rate at 3 months post-procedure was $8.7 \%(2 / 23)$, and mortality rate 3 months post-procedure was $0 \%$. Angiographic follow-up examination was carried out in 10 patients at 6 months and revealed no filling $(0 \%)$ of aneurysm in four patients, entry remnant $(<5 \%)$ in five, and subtotal filling (5\%-95\%) in another patient.

Conclusion: The PED placement is effective therapy for the treatment of large/giant and wide-necked intracranial aneurysms. Despite the relatively low rate of permanent complications associated with the PED, it is important to note that the total complication rate was not negligible.

Keywords \ flow diverter, intracranial aneurysm, Pipeline

\section{Introduction}

Traditional endovascular methods for the treatment of large/giant and wide-necked intracranial aneurysms include a reconstructive approach in which the aneurysm sac is

Department of Neurosurgery, Dokkyo Medical University Koshigaya Hospital, Koshigaya, Saitama, Japan

Received: December 20, 2016; Accepted: March 8, 2017 Corresponding author: Ryuta Nakae. Department of Neurosurgery, Dokkyo Medical University Koshigaya Hospital, 2-1-50 Minamikoshigaya, Koshigaya, Saitama 343-8555, Japan Email:nakae@nms.ac.jp

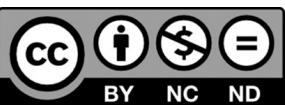

This work is licensed under a Creative Commons Attribution-NonCommercialNoDerivatives International License.

(C)2017 The Japanese Society for Neuroendovascular Therapy filled with detachable platinum coils and parent artery occlusion. However, coil embolization of large/giant and wide-necked intracranial aneurysms has been associated with low rates of initial angiographic occlusion and high rates of recurrence, ${ }^{1,2)}$ and ischemic stroke and formation of de novo aneurysms are well recognized as complications following parent artery occlusion. ${ }^{3,4)}$ Even the adoption of advanced coiling techniques, such as adjunctive balloons, stents, and complex-shaped and biologically enhanced coils, has not improved the outcomes of these methods..$^{5-7)}$

Recently, flow diverters (FDs) have become an attractive alternative for the treatment of intracranial aneurysms. ${ }^{8,9}$ ) These low-porosity stents are placed within the parent artery to direct blood flow away from the aneurysm ${ }^{10)}$ and to promote thrombosis of the aneurysm and neointimal overgrowth of the aneurysm neck. ${ }^{11)}$ This treatment option is 
particularly suited to large/giant, wide-necked, and fusiform aneurysms. ${ }^{8)}$

Following Japanese government approval of the Pipeline Embolization Device (PED) (Covidien, Irvine, CA, USA), a type of FD, in March 2015, the PED became available in selected institutions, including our own. Here, we describe our initial experience with the PED for the treatment of large/giant and wide-necked intracranial aneurysms of the internal carotid artery (ICA) and our findings on its safety, complications, and effectiveness.

\section{Materials and Methods}

\section{Patients}

We retrospectively reviewed the demographic, clinical, and radiological data of all patients who received PED placement at our hospital from November 2015 to August 2016. Patients' cases were included if they had presented with an unruptured intracranial aneurysm arising from the ICA between the petrous and superior hypophyseal segments and proximal to the posterior communicating segment that measured at least $10 \mathrm{~mm}$ in maximum diameter and had a neck measuring at least $4 \mathrm{~mm}$. Patients with recanalized aneurysms needing retreatment after previous embolization were also included. From November 2015 to August 2016, 23 patients were treated with the PED. We recorded patient age and sex, and aneurysm location, maximum diameter, neck diameter, the use of balloon angioplasty for the PED, and instances of coil embolization. Patients provided written consent after they were informed of the treatment's risks and benefits. This study was approved by the hospital's Institutional Review Board.

\section{Assessment}

Before PED placement, all patients underwent a baseline neurological examination, which included detailed neurological and neuro-ophthalmological assessments to evaluate visual acuity (VA), and to identify any neurological deficits, cranial nerve $(\mathrm{CN})$ palsies, and/or visual field (VF) deficits. Patients underwent a repeat examination after PED placement and were scheduled for mandatory clinical follow-up at 30 days and 3 months post-procedure. Modified Rankin scale (mRS) scores were scheduled $(0=$ asymptomatic; $6=$ death) at admission and were independently confirmed by the study's neurointensivists (A.H. and Y.T.) at 30 days and 3 months post-procedure using in-person contact and mail correspondence with the location to which patients were transferred after discharge from our hospital.
All patients underwent high-resolution CT scans just after treatment to evaluate PED placement. MRI scans were routinely obtained 24-36 hours after treatment and scheduled for the 3-month follow-up and as needed, to evaluate neurological deficits.

The procedural efficacy (degree of occlusion) for aneurysms in the 10 patients to reach the 6-month post-procedure point was assessed by DSA using the technique proposed by O'Kelly and Marotta (OKM) grading scale. ${ }^{12)}$ The results were categorized as Grade A: total filling of the aneurysm with contrast (>95\%), Grade B: subtotal filling (5\%-95\%), Grade C: entry remnant ( $<5 \%)$, and Grade D: no filling $(0 \%)$. Findings such as stent migration, in-stent stenosis, and stent occlusion were also recorded.

All patients were pretreated with aspirin (100 mg per day orally for 7 days) and clopidogrel (75 mg per day orally for 7 days). Aspirin reaction units (ARU) and P2Y12 reaction units (PRU) were measured using the rapid platelet function assay Verify Now (Accumetrics, San Diego, CA, USA) to assess aspirin and clopidogrel resistance. When resistance occurred, the aspirin or clopidogrel dose was increased, or cilostazol (200 mg per day) was prescribed. Patients continued clopidogrel therapy for a minimum of 6 months, and aspirin therapy for a minimum of 1 year, after treatment.

\section{Technique}

The procedures were performed using coaxial endovascular navigation under general anesthesia. Following arterial access, patients were typically administered intravenous heparin to achieve an activated clotting time of $250 \mathrm{sec}-$ onds. A 7 Fr guiding sheath (Shuttle Sheath, Cook Medical, Bloomington, IN, USA) was placed into the proximal cervical segment of the ICA. A 6 or 5 Fr distal support catheter (Navien, Covidien) was placed into the proximal intracranial segment of the ICA and 0.027-inch inner diameter delivery microcatheter (Marksman, Covidien) was placed into position across the aneurysm neck over a 0.014-inch outer diameter microguidewire (CHIKAI, Asahi Intecc, Nagoya, Japan). When coiling was performed in conjunction with PED treatment, another microcatheter was navigated "jailed" into the aneurysm alongside the delivery microcatheter within the parent artery. After positioning of the delivery microcatheter, the PED was loaded into the delivery microcatheter hub using a rotating hemostatic valve and advanced using the delivery wire. The PED was then deployed in the targeted landing zone via a process involving unsheathing of the delivery microcatheter and stabilization and advancement of the delivery 
wire. Following deployment, the delivery microcatheter was advanced over the delivery wire to the PED distal edge. Once the PED was in place, if balloon angioplasty for expansion of the PED was to be performed, the delivery wire was changed to a 0.010 -inch outer diameter, $300 \mathrm{~cm}$ long microguidewire (X-Celerator, Covidien), and the delivery microcatheter was exchanged for a micro-balloon catheter (HyperForm, Covidien). Following placement of the PED, adjunctive coils were used via the jailed microcatheter when there was high inflow into the intracranial aneurysm, which is thought to be a risk factor of postprocedural rupture. ${ }^{13)}$ After coil embolization, the coil microcatheter was gently removed from the aneurysm without displacement of the construct.

\section{Results}

\section{Patient population}

Over the course of 10 months, 23 intracranial aneurysms in 23 consecutive patients ( 1 male and 22 females) with a median age of 66 (61-72) years (25th-75th percentiles) were treated using the PED (Table 1). Reasons for treatment included aneurysms incidentally found in 12 patients $(52.2 \%)$, recurrence of previously embolized aneurysms in four patients $(17.4 \%)$, aneurysms discovered because of diplopia in four patients (17.4\%), VA loss in two patients (8.7\%), and VF defect in one patient (4.3\%).

\section{Aneurysm characteristics}

In all, 23 aneurysms were treated; 22 were saccular (95.7\%), and 1 was fusiform (4.3\%). In all, 11 saccular aneurysms (47.8\%) were located in the cavernous segment of the ICA, whereas the other $11(47.8 \%)$ were found on the paraclinoid segment (seven on the infraclinoid segment and four on the supraclinoid segment). The fusiform aneurysm extended from the petrous to the infraclinoid segment of the ICA. Three aneurysms (13.1\%) were medium (10-12 mm), 17 (73.8\%) were large (12-24 mm), and $3(13.1 \%)$ were giant size $(>24 \mathrm{~mm})$. All saccular aneurysms were wide-necked, and mean neck diameter was $7.5 \pm 2.6 \mathrm{~mm}$ (standard deviation). The fusiform aneurysm had no distinct neck so neck diameter could not be evaluated (Table 2).

\section{Treatment and technical outcomes}

In all, 22 aneurysms were treated using one PED each, and one using two PEDs in two separate procedures due to coil compaction in Case 13/13A (Table 1). For 20 aneurysms
(87.0\%), a PED alone was used, while for 3 aneurysms $(13.0 \%)$ with high inflow, PED and coils were used. Adjunctive balloon angioplasty using a HyperForm balloon system was needed for proper apposition of the stent against the ICA wall in 14 cases (60.9\%).

\section{Complications}

A total of 11 complications were experienced by nine patients with a mean follow-up period of 6.5 months (Table 1). Two procedure-related symptomatic ischemic strokes $(8.7 \%)$ confirmed by brain MRI with diffusionweighted imaging (DWI) occurred. Both had resolved completely by post-procedure day 7 . We observed new neuro-ophthalmological deficits in five patients (21.7\%): four presented with CN III palsy and one with VA deficit. Three of the CN III palsies and the VA deficit had resolved completely by post-procedure day 18 ; the other patient with CN III palsy showed completely improvement by 3 months post-procedure. One patient (4.3\%, Case 13$)$ had symptomatic cerebral edema, probably due to mass effect from aneurysmal volume expansion after PED placement. Coil compaction was observed in Case 13, and retreatment using an overlapping PED was performed at 3 months after the first operation. One patient $(4.3 \%)$ presented with delayed ischemic stroke on post-procedure day 14 , and two $(8.7 \%)$ with delayed intracerebral hemorrhages (ICH) on post-procedure days 6 and 15. Transient CN III palsy, delayed ischemic stroke, and delayed ICH occurred in one patient. One patient with ICH was asymptomatic and the other showed severe hemiparesis and moderate motor aphasia. Overall, the total complication rate was 39.1\% (9/23) with using the PED. Morbidity rate at 3 months post-procedure was $8.7 \%(2 / 23)$ and mortality rate at 3 months post-procedure was $0 \%(0 / 23)$.

\section{Clinical outcomes}

In two patients, mRS had worsened at 3 months postprocedure (Cases 8 and 13). One patient with diplopia before treatment showed complete improvement by 3 months post-procedure. Other three patients with diplopia and one patient with VF defect before treatment noted moderate improvement in their symptoms although their mRS scores had not changed at 3 months (Table 1).

\section{Angiography findings at 6 months}

DSA was performed in 10 patients to determine the status of their aneurysms at 6 months post-procedure (Table 2). At that time, there were four OKM Grade D aneurysms, 


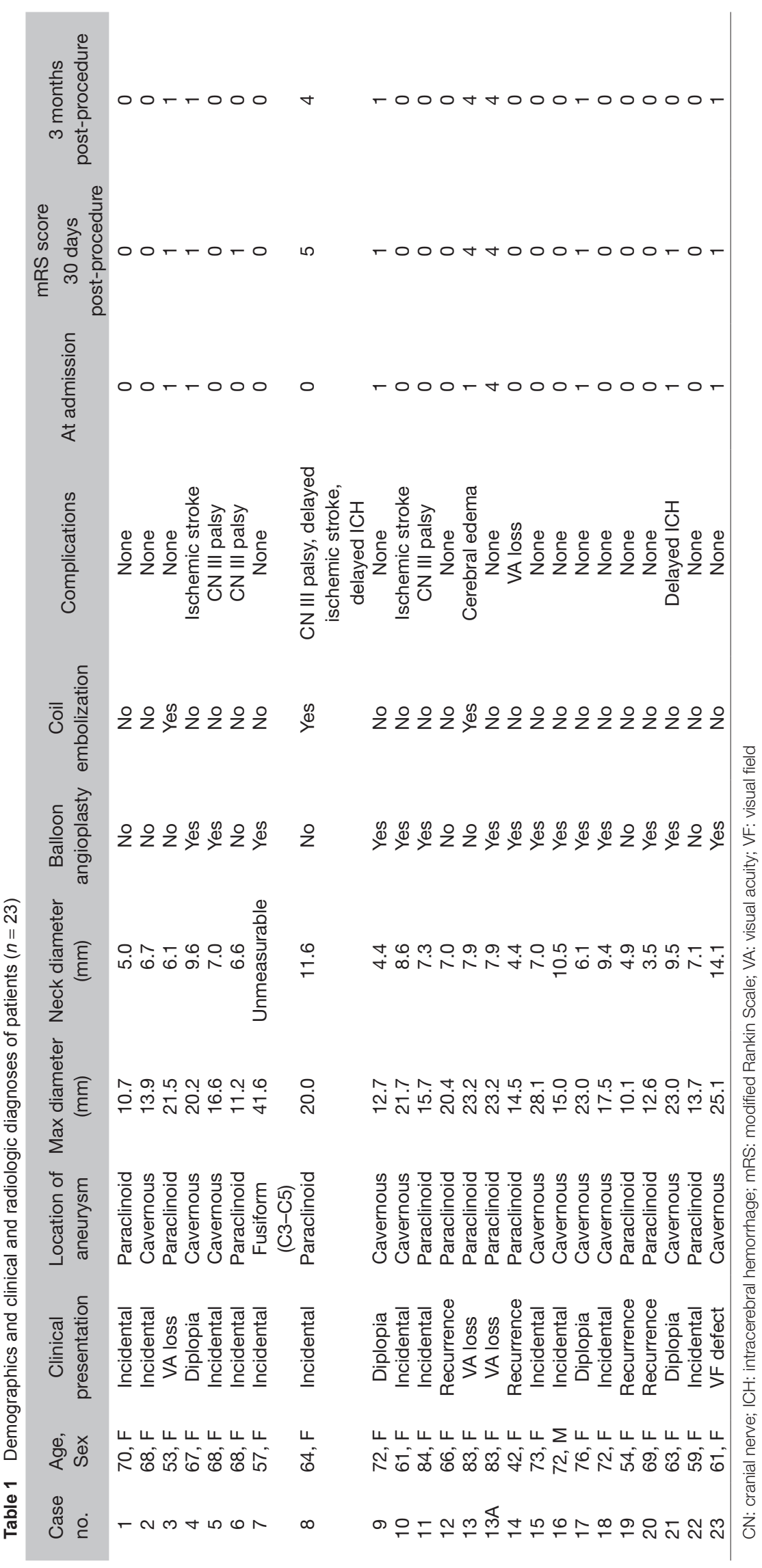


Table 2 Angiography findings at 6 months post-procedure $(n=10)$

\begin{tabular}{rrrrr} 
Case no. & OKM grading scale & Stent migration & In-stent stenosis & Stent occlusion \\
1 & Grade D & No & No & No \\
2 & Grade D & No & No & No \\
3 & Grade C & No & No & No \\
4 & Grade C & No & No & No \\
5 & Grade C & No & No & No \\
6 & Grade D & No & No & No \\
7 & Grade B & No & No & No \\
10 & Grade C & No & No & No \\
\hline
\end{tabular}

Grade A: total filling of the aneurysm with contrast (>95\%); Grade B: subtotal filling (5\%-95\%); Grade C: entry remnant (<5\%); Grade D: no filling (0\%); OKM: O'Kelly and Marotta

five OKM Grade C aneurysm, and one OKM Grade B aneurysm. Stent migration, in-stent stenosis, or stent occlusion was not detected in any of the nine patients.

\section{Illustrative cases}

Case 2: A 68-year-old woman presented with an incidentally found intracranial aneurysm measuring $13.9 \times$ $7.1 \mathrm{~mm}$, located in the cavernous segment of the left ICA (Fig. 1). Endovascular treatment was performed with a single PED $(4.0 \times 16 \mathrm{~mm})$, which was deployed across the neck of the aneurysm; no endosaccular coils were used. She was discharged 11 days after the operation with no neurological deficits. A 6-month follow-up DSA revealed complete occlusion (OKM Grade D) of her lesion and she remains neurologically intact.

Case 6: A 68-year-old woman presented with an incidentally found intracranial aneurysm measuring $11.2 \times 8.4 \mathrm{~mm}$, located in the infraclinoid segment of the left ICA (Fig. 2). Endovascular treatment was performed using a single PED $(4.25 \times 20 \mathrm{~mm})$, which was deployed across the aneurysm neck without the use of endosaccular coils. Immediately after the procedure, CN III palsy (ptosis, mydriasis, and diplopia) was observed. Brain MRI and MRA did not reveal complications in the brain, and the left ICA was patent. We compared the aneurysmal sizes pre- and post-procedure using MRA with time-of-flight (TOF) source imaging, and found no difference between them. She was treated with corticosteroids. Mydriasis and diplopia were completely resolved by 2 months post-procedure and ptosis was completely resolved by 3 months post-procedure. A 6-month follow-up DSA revealed complete occlusion (OKM Grade D) of her lesion.

Case 8: A 64-year-old woman presented with an incidentally found intracranial aneurysm measuring $20.0 \times$ $18.5 \mathrm{~mm}$, located in the infraclinoid segment of the left ICA (Fig. 3). Endovascular treatment was conducted using a single PED $(4.0 \times 20 \mathrm{~mm})$, which was deployed across the aneurysm neck and endosaccular coils were used. On post-procedure day 4, CN III palsy (ptosis) was observed. Brain MRI and MRA did not reveal a complication in the brain, and the left ICA was patent. She was treated with corticosteroids and symptoms had completely disappeared by post-procedure day 11 . She was discharged 11 days after the procedure with no neurological deficit. Three days after discharge, the patient was admitted to our hospital with the sudden onset of moderate right hemiparesis and mild motor aphasia. MRI with DWI revealed acute cerebral infarction in the left frontal, temporal, and parietal lobes. In-stent thrombosis was suspected and heparin was administered to achieve an activated clotting time of 250 seconds. After 24 hours, the patient's consciousness level suddenly decreased. Brain CT scans revealed a $70 \times$ $45 \mathrm{~mm}$ ICH in the infarcted area noted on DWI with intraventricular hemorrhage and midline shift. Craniotomy and evacuation of the hematoma were performed, resulting in improvements in her level of consciousness but severe right hemiparesis and moderate motor aphasia persisted. She was transferred to the rehabilitation hospital 76 days after the second admission.

Case 21: A 63-year-old woman was referred to our hospital for treatment of an intracranial aneurysm measuring $23.0 \times 14.4 \mathrm{~mm}$, located in the cavernous segment of the right ICA, which had been found during investigation of her diplopia (Fig. 4). Endovascular treatment was performed with a single PED $(4.0 \times 25 \mathrm{~mm})$, which was deployed across the neck of the aneurysm; no endosaccular coils were used. Brain CT scans at 6 days after procedure revealed a $10 \times 7 \mathrm{~mm}$ asymptomatic $\mathrm{ICH}$ in the right parietal lobe that did not correspond to a hyperintense lesion on post-procedural DWI. Conservative therapy was performed and she was discharged 21 days after the operation with mild improvement in her diplopia. 

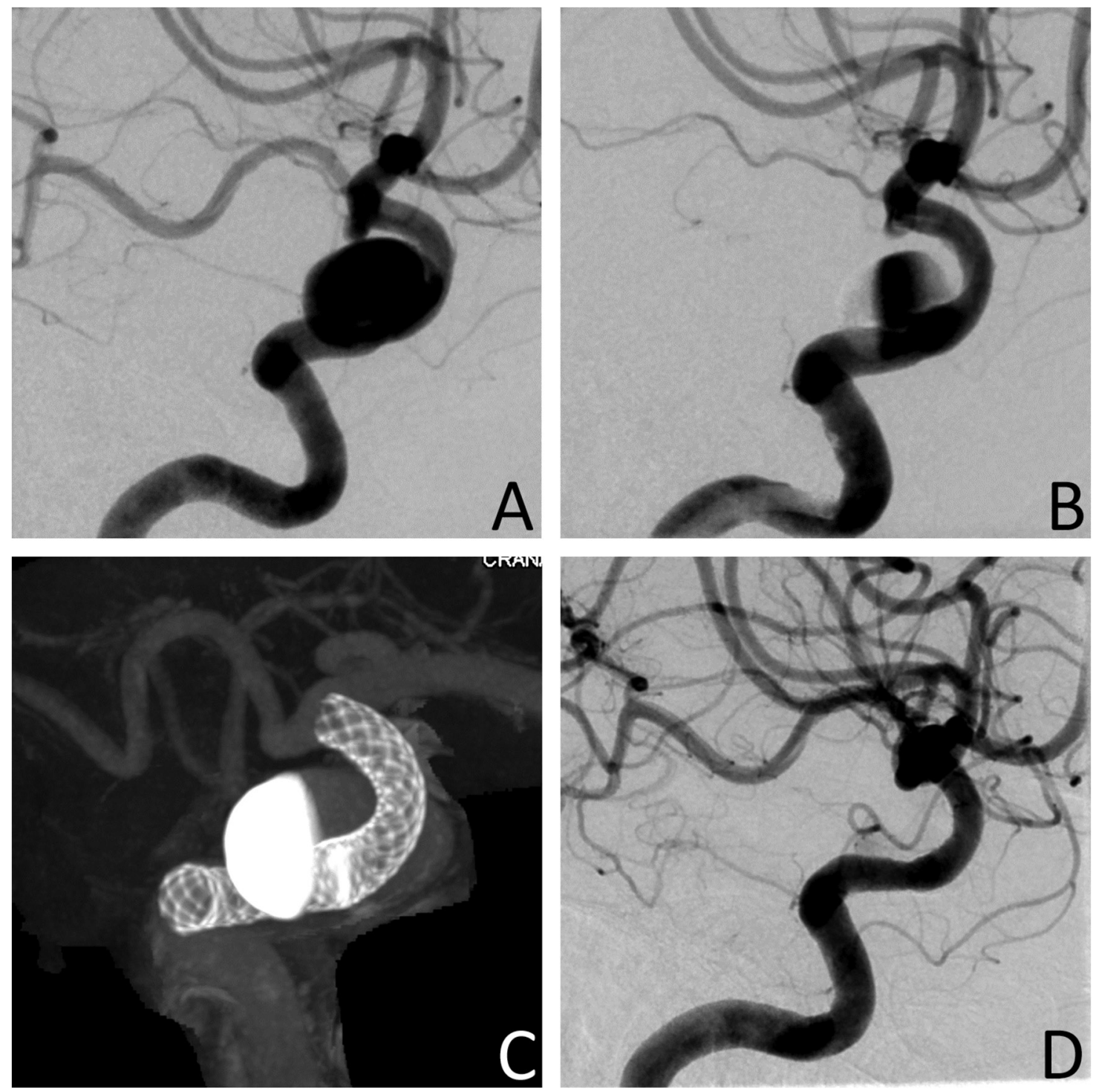

Fig. 1 Case 2: (A) Postero-lateral view of DSA revealed a large intracranial aneurysm measuring $13.9 \times 7.1 \mathrm{~mm}$, located in the cavernous segment of the left internal carotid artery. (B) Postero-lateral view of DSA obtained post-PED placement revealed a reduced flow into the aneurysm dome in the arterial phase. (C) Multiplanar reconstructed dyna-computed tomography imaging obtained post-procedure with dilute contrast injection enabled visualization of the deployed PED covering the neck of the aneurysm. (D) Postero-lateral view DSA obtained 6 months post-procedure revealed complete thrombosis of the aneurysm. PED: Pipeline Embolization Device

\section{Discussion}

FDs have been used to successfully treat complex aneurysms that could not previously be resolved by endovascular treatment. Several studies have reported a favorable safety and efficacy profile. A systematic review of 10 publications by Leung et al. ${ }^{14)}$ involving PED use in 414 patients with 448 intracranial aneurysms identified an overall procedural complication rate of $10.3 \%$, an intracranial vascular complication rate of $6.3 \%$, and procedural mortality rate of $2.2 \%$. A larger meta-analysis of 29 studies performed by Brinjikji et al. ${ }^{15}$ ) including 1451 patients with 1654 aneurysms noted a procedure-related morbidity rate of $5.0 \%$ and mortality rate of $4.0 \%$. An international retrospective study of the PED by Kallmes et al. ${ }^{16)}$ involving PED use in 263 unruptured aneurysms of at least $10 \mathrm{~mm}$ in maximum diameter arising from the ICA identified a neurologic morbidity and mortality rate of $7.4 \%$ and $3.8 \%$, respectively. Park et al. ${ }^{17)}$ reported a total complication rate associated with PED use of $31.7 \%$ (40/126), which was congruent to our results. Four complications (3.2\%) were permanent and the other $36(28.6 \%)$ were reversible. 

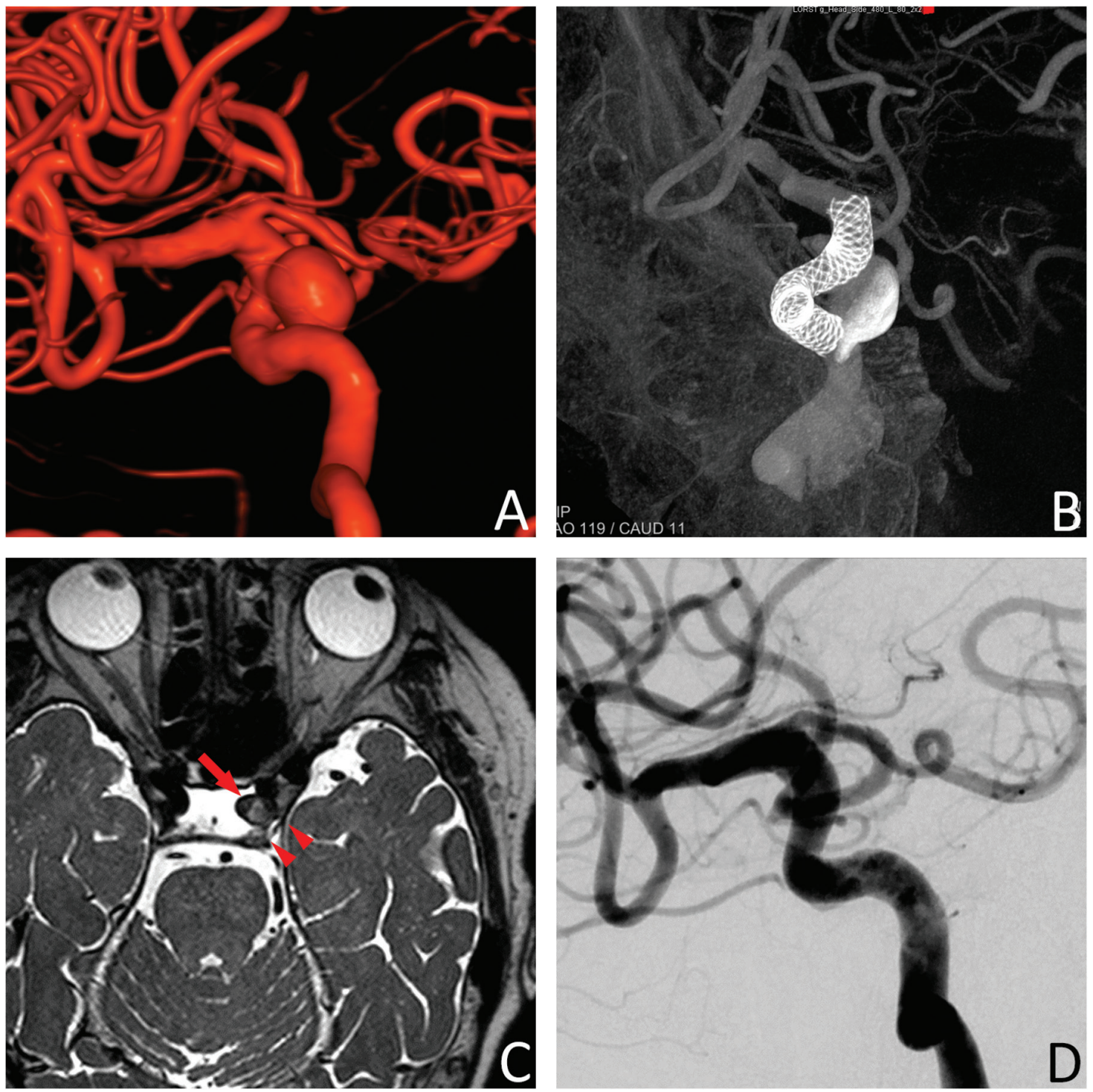

Fig. 2 Case 6: (A) Postero-lateral view of three-dimensional rotational angiography revealed a large intracranial aneurysm measuring $11.2 \times 8.4 \mathrm{~mm}$, located in the infraclinoid segment of the left internal carotid artery. (B) Multiplanar reconstructed dynamic computed tomography imaging obtained post-procedure with dilute contrast injection enabled visualization of the deployed Pipeline Embolization Device covering the neck of the aneurysm. (C) Magnetic resonance imaging with CISS revealed positional relation of intracranial aneurysm (arrow) and cranial nerve III (arrowheads). The aneurysm was in contact with cranial nerve III. (D) Postero-lateral view of DSA obtained 6 months post-procedure revealed complete thrombosis of the aneurysm. CISS: constructive interference in steady state

Our study demonstrated that $8.7 \%$ of patients suffered procedure-related acute ischemic strokes over a 6.5 -month mean follow-up interval, but these were not lethal. Leung et al. ${ }^{14)}$ demonstrated a symptomatic ischemic rate of $3.1 \%$. Brinjikji et al. ${ }^{15)}$ noted an acute ischemic stroke rate of $5.0 \%$ within 30 days of FD treatment and 3.0\% beyond 30 days. Iosif et al. ${ }^{18)}$ showed that treatment with PED resulted in at least one new hyperintense lesion on postprocedural DWI in 35 of 38 consecutive patients (92.1\%) within 48 hours post-procedure. Overall, 33 patients $(86.8 \%)$ were free of clinical complications during the first 3 months post-procedure, while five (13.2\%) experienced new neurological symptoms, with the symptoms in three $(7.9 \%)$ being irreversible. Lesion numbers are significantly higher when flow-diverting stents are used, even in comparison with stent-assisted coiling, ${ }^{19)}$ revealing an increased embolic tendency associated with these devices, possibly due to its mechanical properties and the technical manipulations required during the procedures. Heller et al. ${ }^{20)}$ reported that the only identifiable risk factor for embolism after use of 

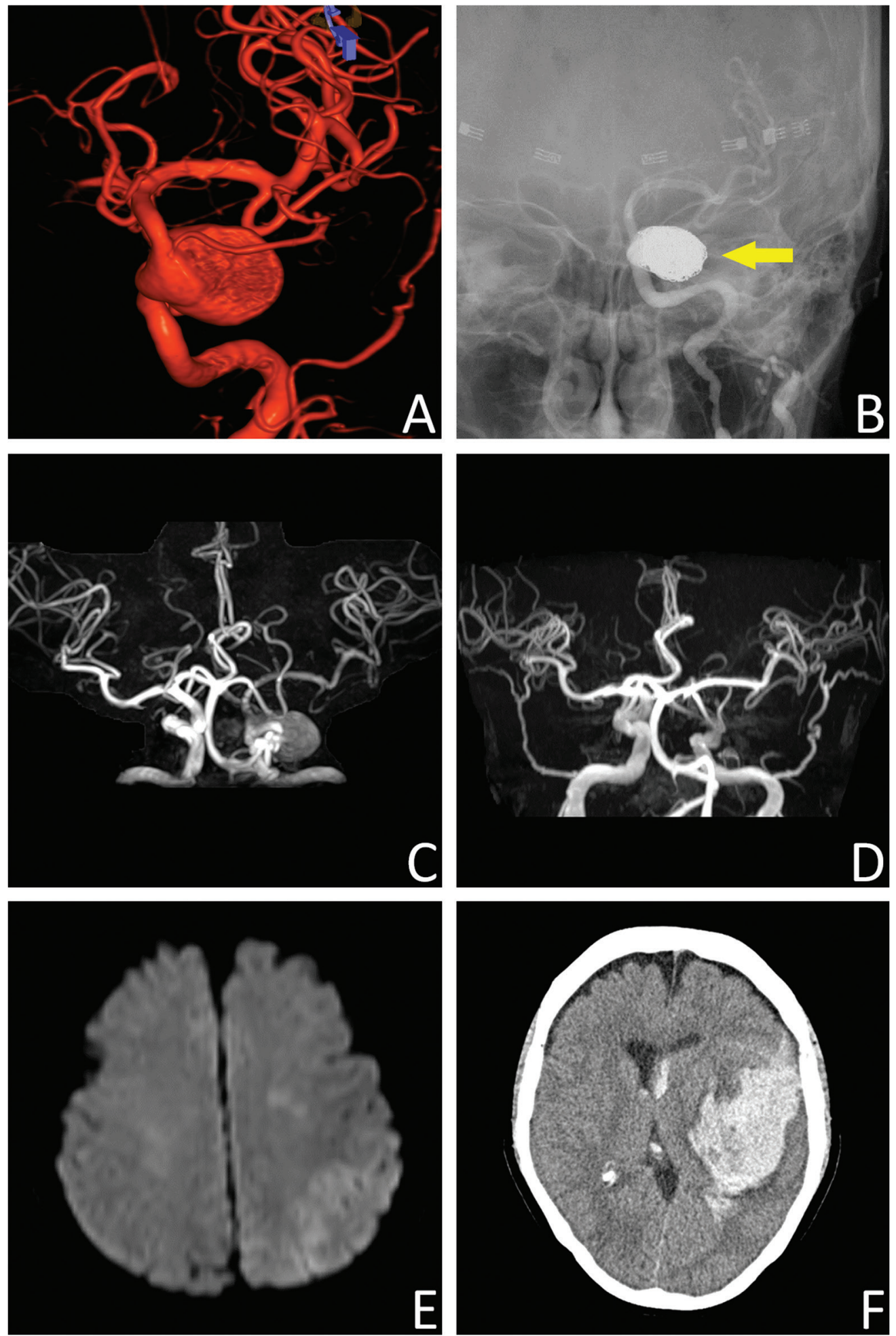

Fig. 3 Case 8: (A) Anterior view of three-dimensional rotational angiography revealed a large intracranial aneurysm measuring $20.0 \times 18.5 \mathrm{~mm}$, located in the infraclinoid segment of the left internal carotid artery. (B) The aneurysm was embolized with eight detachable coils (arrow) in conjunction with PED treatment. (C) Brain MRA obtained before PED placement. (D) Brain MRA obtained post-procedure day 1 revealed an increase in flow signal in the left middle cerebral artery. (E) Brain magnetic resonance imaging with DWI obtained postprocedure day 14 revealed acute cerebral infarction in the left frontal and parietal lobes. (F) Brain computed tomography obtained post-procedure day 15 revealed a $70 \times 45 \mathrm{~mm}$ intracerebral hemorrhage in the same infarcted area noted on DWI with intraventricular hemorrhage and midline shift. DWI: diffusion-weighted imaging; PED: Pipeline Embolization Device 

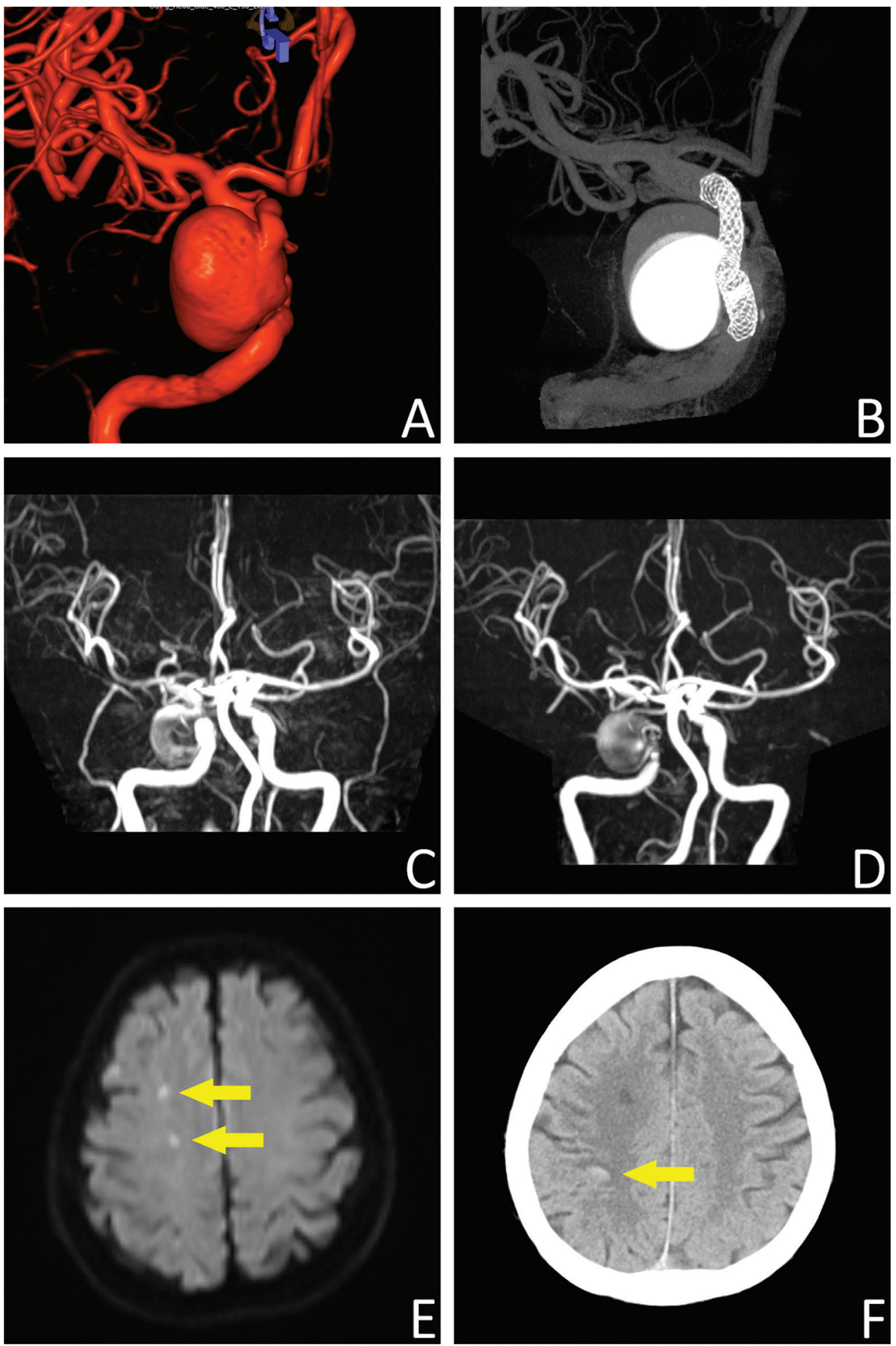

Fig. 4 Case 21: (A) Anterior view of three-dimensional rotational angiography revealed a large intracranial aneurysm measuring $23.0 \times 14.4 \mathrm{~mm}$, located in the cavernous segment of the right internal carotid artery. (B) Multiplanar reconstructed dynamic computed tomography imaging obtained post-procedure with dilute contrast injection enabled visualization of the deployed PED traversing the neck of the contrast-filled aneurysm. (C) Brain MRA obtained before PED placement. (D) Brain MRA obtained post-procedure day 1 revealed an increase in flow signal in the right middle cerebral artery. (E) Brain magnetic resonance imaging with DWI obtained post-procedure day 1 revealed hyperintense lesions in the right frontal lobe (arrows). (F) Brain computed tomography obtained post-procedure day 6 revealed a $10 \times 7 \mathrm{~mm}$ intracerebral hemorrhage in the right parietal area (arrow), separate from the hyperintense lesions seen on post-procedural DWI. DWI: diffusion-weighted imaging; PED: Pipeline Embolization Device 
PEDs is an increase in aneurysm size, possibly indicating to a disturbance in the flow across the aneurysm neck with ingress and egress through the PED struts. Regardless of their nature, endovascular procedures appear to have a tendency to produce microembolic events. The majority of these remains silent following appropriate anti-aggregation treatment and technique compliance, and do not appear to impact the safety of the procedure.

Mass or flow-related dynamic effects from large and giant aneurysms of the ICA between the petrous and supraclinoid segments may cause cranial neuropathies that affect CN III, IV, V, and VI. ${ }^{21)}$ In addition, optic neuropathy may develop from direct compression by large and giant aneurysms, resulting in VA and VF deficits. ${ }^{21)}$ The Pipeline for Uncoilable or Failed Aneurysms (PUFS) trial ${ }^{21}$ observed a neuro-ophthalmological baseline deficit in 39 of 98 patients $(39.8 \%)$ that was presumed attributable to the intracranial aneurysm, with a higher proportion of patients having aneurysms of the cavernous segment (57\%) than of the petrous $(0 \%)$, superior hypophyseal (22\%), paraophthalmic (27\%), or supraclinoid (25\%) segments. Moreover, they observed single new deficits in 5 of 98 patients (5.1\%) at 6 months post-procedure: three with CN VI palsy, one with $\mathrm{CN}$ IV palsy, and one with a VA deficit. The maximum aneurysmal diameter was significantly larger in patients with a new deficit or an exacerbated baseline deficit at 6 months postprocedure. Brown et al. ${ }^{22)}$ reported that baseline $\mathrm{CN}$ palsies worsened in the short term after placement of FDs in $15.6 \%$ of their patients, but decreased to $4.8 \%$ by 12 months post-procedure. Meanwhile, the mechanism by which new $\mathrm{CN}$ deficits occur after PED placement is uncertain. Different reports have proposed three main causes of post-procedure $\mathrm{CN}$ deficits: mass effect due to aneurysmal volume expansion immediately after FD-induced thrombosis, intra-aneurysmal hemodynamic alterations caused by FDs, and perianeurysmal inflammation linked to FD-induced thrombosis.

Volume expansion after endovascular treatment of large and giant intracranial aneurysms is thought to be secondary to thrombus formation within the aneurysm sac, induced from coil embolization and/or FDs. ${ }^{23)}$ Carneiro et al. ${ }^{23)}$ reported that after eight FD deployments, two aneurysms had increased in size, three were unchanged, and three decreased in volume after a mean follow-up period of 20 months. There are currently no reports about aneurysmal volume expansion immediately after PED placement. We suggest that slight aneurysmal volume expansion below the resolution of imaging and subsequent contraction due to FD-induced thrombosis may occur.
Intra-aneurysmal hemodynamic alterations are the other proposed cause of $\mathrm{CN}$ deficits after PED placement. Larrabide et al. ${ }^{24}$ used computational fluid dynamics (CFD) to show that FD treatment significantly increased the flow pulsatility in aneurysms. Hassan et al. ${ }^{25)}$ also used CFD models of aneurysms to show that, until aneurysms are completely healed, the wall of the aneurysm is subject to stresses and strains from the pulsatile arterial circulation, with higher intra-aneurysmal pressure imposed by the FDs. A decrease in blood flow does not equate to a decrease in the pressure on the wall of the aneurysm.

Perianeurysmal inflammation linked to FD-induced thrombosis has also been documented. Berge et al. ${ }^{26)}$ reported a high frequency of perianeurysmal inflammation after treatment of unruptured aneurysms with FDs. These presented as perianeurysmal brain edemas, shown by high signal intensity on FLAIR MRI images. Risk factors associated with perianeurysmal inflammation were large aneurysm size and close contact with adjacent parenchyma. Stutz et al. ${ }^{27)}$ reported that the death of endothelial cells that line the wall of the aneurysm is associated with massive thrombosis and leads to perilesional cortical inflammation. Endothelial vascularization of the aneurysm sac is compromised by thrombosis, and results in anoxia/ischemia of the endothelium wall, cell death, and the release of stress signals, collectively termed "damage-associated molecular patterns." We suggest that perianeurysmal inflammation affects not only the parenchyma but also CNs.

No patients have experienced permanent $\mathrm{CN}$ palsy in our series. New neuro-ophthalmological deficits occurred in five patients, four of which had completely resolved by post-procedure day 18 and the other patient showed completely improvement by 3 months post-procedure.

Recently, several reports have demonstrated that delayed ipsilateral ICH is a potential complication of flow diversion treatment of intracranial aneurysms. ${ }^{28,29)}$ These reports all showed that patients awoke from general anesthesia in a baseline condition, but subsequently developed delayed neurological deterioration concerning the development of ICH in vasculature ipsilateral but distal to the treated aneurysm at hours to days post-procedure. In the PUFS trial, $\left.{ }^{8}\right)$ ICH occurred in 5 of 107 patients (4.7\%), and was attributed to ischemia, trauma, and warfarin use. The PED for the Intracranial Treatment of Aneurysms (PITA) trial ${ }^{19}$ demonstrated no delayed ICH in any of the 31 patients treated with the device. These two trials did not require routine postoperative brain imaging, so minor or asymptomatic ICH may not have been detected. Brinjikji et al. ${ }^{15)}$ noted an ICH 
rate of $3.0 \%$ within 30 days of FD treatment and $2.0 \%$ beyond 30 days. The pathophysiology of this condition is unknown. One proposed hypothesis for this delayed ipsilateral ICH is that replacement of the relatively compliant wall segment with a more rigid stent changes pressure transmission across the segment, ${ }^{28,29)}$ causing a reduction in the so-called Windkessel effect, ${ }^{29)}$ which describes a decrease in blood vessel elasticity that results in increased distal pulse pressure. Chiu and Wenderoth ${ }^{30)}$ postulated that cerebral hyperperfusion syndrome can occur following FD placement across an aneurysm neck. In their case report, the authors suggested that by diverting blood flow into the parent vessel from the aneurysm sac and reducing aneurysm expansion during systole, FDs increase perfusion to the distal arterial territory, which can cause cerebral hyperperfusion syndrome due to a reduction in the Windkessel effect. In contrast, other researchers have proposed that the pathophysiology involves an intra-procedural or post-procedural infarct, possibly resulting from thrombus formation on the stent and subsequent embolism. ${ }^{28,29)}$ Brinjikji et al. ${ }^{28)}$ reported that ICH after PED placement increased fourfold following multiple PEDs due to secondary hemorrhagic transformation of the resulting ischemic infarcts, which would reflect a much greater metal surface area exposed to platelets. In the present study, ICH in Case 8 was recognized in the same infarcted area noted on DWI, whereas ICH in Case 21 occurred in another area separate from the post-procedure infarction. To compare MRA obtained before and after PED placement in cases 8 and 21, MRA obtained post-procedure on day 1 revealed an increase in flow signal in the ipsilateral middle cerebral artery, reflecting a reduction in the Windkessel effect. An idiopathic hemorrhagic infarction and/or an increase in distal pulse pressure from reduction in the Windkessel effect was thought to be the cause of delayed ICH in our cases.

In their systematic review, Leung et al. ${ }^{14)}$ noted that eight studies reported angiographic follow-up at 6 months or beyond for a total of 354 intracranial aneurysms, and that complete obliteration was achieved in 293 lesions (82.8\%). A larger meta-analysis by Brinjikji et al. ${ }^{15}$ reported a complete occlusion rate of $76 \%$ at 6 months. In the present study, there were 9 out of $10(90.0 \%)$ aneurysms of which a 6-month follow-up DSA revealed $>95 \%$ occlusion. In contrast, our complete occlusion rate of $40.0 \%$ differs from previous reports, probably due to the small sample size. We await long-term radiographic results.

Several limitations of the study warrant mention. Our study was composed of 23 patients harboring 23 aneurysms.
This was relatively small and long-term clinical and radiographic results are lacking. Further investigation and implementation of long-term follow-up studies of these patients is necessary.

\section{Conclusion}

PED is a feasible and effective method for large/giant and wide-necked intracranial aneurysms. However, the overall complication rate is not negligible although the rate of permanent morbidity and mortality rate was low. The main complications of the PED included ischemic stroke, CN deficits, and delayed ipsilateral ICH. Future studies to identify factors that predispose patients to these complications are necessary.

\section{Disclosure Statement}

The authors have no personal financial or institutional interest in any of the drugs, materials, or devices described in this article.

\section{References}

1) Campi A, Ramzi N, Molyneux AJ, et al: Retreatment of ruptured cerebral aneurysms in patients randomized by coiling or clipping in the international subarachnoid aneurysm Trial (ISAT). Stroke 2007; 38: 1538-144.

2) Raymond J, Guilbert F, Weill A, et al: Long-term angiographic recurrences after selective endovascular treatment of aneurysms with detachable coils. Stroke 2003; 34: 1398-1403.

3) Dyste GN, Beck DW: De novo aneurysm formation following carotid ligation: case report and review of the literature. Neurosurgery 1989; 24: 88-92.

4) Larson JJ, Tew JM Jr, Tomsick TA, et al: Treatment of aneurysms of the internal carotid artery by intravascular balloon occlusion: long-term follow-up of 58 patients: 23 . Neurosurgery 1995; 36: 26-30.

5) Geyik S, Ertugrul O, Yavuz K, et al: Comparison of bioactive coils and bare platinum coils for treatment of intracranial aneurysms: a matched-pair analysis. J Neurosurg 2010; 112: 709-713.

6) Pierot L, Leclerc X, Bonafé A, et al: Endovascular treatment of intracranial aneurysms with matrix detachable coils: midterm anatomic follow-up from a prospective multicenter registry. AJNR Am J Neuroradiol 2008; 29: 57-61.

7) Shapiro M, Becske T, Sahlein D, et al: Stent-supported aneurysm coiling: a literature survey of treatment and followup. AJNR Am J Neuroradiol 2012; 33: 159-163. 
8) Becske T, Kallmes DF, Saatci I, et al: Pipeline for uncoilable or failed aneurysms: results from a multicenter clinical trial. Radiology 2013; 267: 858-868.

9) Nelson PK, Lylyk P, Szikora I, et al: The pipeline embolization device for the intracranial treatment of aneurysms trial. AJNR Am J Neuroradiol 2011; 32: 34-40.

10) Lieber BB, Stancampiano AP, Wakhloo AK: Alteration of hemodynamics in aneurysm models by stenting: influence of stent porosity. Ann Biomed Eng 1997; 25: 460-469.

11) Kallmes DF, Ding YH, Dai D, et al: A second-generation, endoluminal, flow-disrupting device for treatment of saccular aneurysms. AJNR Am J Neuroradiol 2009; 30: 1153-1158.

12) O'kelly CJ, Krings T, Fiorella D, et al: A novel grading scale for the angiographic assessment of intracranial aneurysms treated using flow diverting stents. Interv Neuroradiol 2010; 16: 133-137.

13) Kulcsár Z, Houdart E, Bonafé A, et al: Intra-aneurysmal thrombosis as a possible cause of delayed aneurysm rupture after flow-diversion treatment. AJNR Am J Neuroradiol 2011; 32: 20-25.

14) Leung GK, Tsang AC, Lui WM: Pipeline embolization device for intracranial aneurysm: a systematic review. Clin Neuroradiol 2012; 22: 295-303.

15) Brinjikji W, Murad MH, Lanzino G, et al: Endovascular treatment of intracranial aneurysms with flow diverters: a meta-analysis. Stroke 2013; 44: 442-447.

16) Kallmes DF, Hanel R, Lopes D, et al: International retrospective study of the pipeline embolization device: a multicenter aneurysm treatment study. AJNR Am J Neuroradiol 2015; 36: 108-115.

17) Park MS, Albuquerque FC, Nanaszko M, et al: Critical assessment of complications associated with use of the pipeline embolization device. $J$ Neurointerv Surg 2015; 7: 652-659.

18) Iosif C, Camilleri Y, Saleme S, et al: Diffusion-weighted imaging-detected ischemic lesions associated with flowdiverting stents in intracranial aneurysms: safety, potential mechanisms, clinical outcome, and concerns. J Neurosurg 2015; 122: 627-636.

19) Hahnemann ML, Ringelstein A, Sandalcioglu IE, et al: Silent embolism after stent-assisted coiling of cerebral aneurysms: diffusion-weighted MRI study of 75 cases. J Neurointerv Surg 2014; 6: 461-465.

20) Heller RS, Dandamudi V, Lanfranchi M, et al: Effect of antiplatelet therapy on thromboembolism after flow diversion with the pipeline embolization device. J Neurosurg 2013; 119: 1603-1610.

21) Sahlein DH, Fouladvand M, Becske T, et al: Neuroophthalmological outcomes associated with use of the pipeline embolization device: analysis of the PUFS trial results. J Neurosurg 2015; 123: 897-905.

22) Brown BL, Lopes D, Miller DA, et al: The fate of cranial neuropathy after flow diversion for carotid aneurysms. J Neurosurg 2016; 124: 1107-1113.

23) Carneiro A, Rane N, Küker W, et al: Volume changes of extremely large and giant intracranial aneurysms after treatment with flow diverter stents. Neuroradiology 2014; 56: 51-58.

24) Larrabide I, Geers AJ, Morales HG, et al: Change in aneurysmal flow pulsatility after flow diverter treatment. Comput Med Imaging Graph 2016; 50: 2-8.

25) Hassan T, Ahmed YM, Hassan AA: The adverse effects of flow-diverter stent-like devices on the flow pattern of saccular intracranial aneurysm models: computational fluid dynamics study. Acta Neurochir (Wien) 2011; 153: 1633-1640.

26) Berge J, Tourdias T, Moreau JF, et al: Perianeurysmal brain inflammation after flow-diversion treatment. AJNR Am J Neuroradiol 2011; 32: 1930-1934.

27) Stutz A, Golenbock DT, Latz E: Inflammasomes: too big to miss. J Clin Invest 2009; 119: 3502-3511.

28) Brinjikji W, Lanzino G, Cloft HJ, et al: Risk factors for hemorrhagic complications following pipeline embolization device treatment of intracranial aneurysms: results from the international retrospective study of the pipeline embolization device. AJNR Am J Neuroradiol 2015; 36: 2308-2313.

29) Cruz JP, Chow M, O'Kelly C, et al: Delayed ipsilateral parenchymal hemorrhage following flow diversion for the treatment of anterior circulation aneurysms. AJNR Am J Neuroradiol 2012; 33: 603-608.

30) Chiu AH, Wenderoth J: Cerebral hyperperfusion after flow diversion of large intracranial aneurysms. J Neurointerv Surg 2013; 5: e48. 